\title{
Cranial neuropathy
}

\author{
OC Cockerell, R Kapoor
}

A 53-year-old woman first presented to her local hospital with anaemia, hypercalcaemia, and bone pain. A bone marrow biopsy showed extensive plasma cell infiltration. She was treated with combination chemotherapy, and later high-dose melphalan and peripheral blood stem cell transplant after total body irradiation. She entered remission and was well until five months later when she awoke with a horizontal diplopia relieved by covering either eye. Two days later she started to develop difficulty chewing food with the right side of her mouth.

On examination she had a right sixth nerve palsy, and a complete sensory and motor trigeminal nerve palsy. She was otherwise well. Investigations, which included a blood film, calcium and serum protein estimation, Bence - Jones urinalysis, and a computed tomographic brain scan, were all normal or negative. Magnetic resonance imaging (MRI) was carried out (figure), followed by a diagnostic procedure.

The National Hospital for Neurology and Neurosurgery, Queen Square, London WC1N 3BG, UK

OC Cockerell

R Kapoor

Correspondence to $\mathrm{Dr}$ Cockerell, Department of Neurology, Guy's Hospital, London SE1 9RT, UK

Accepted 1 May 1996

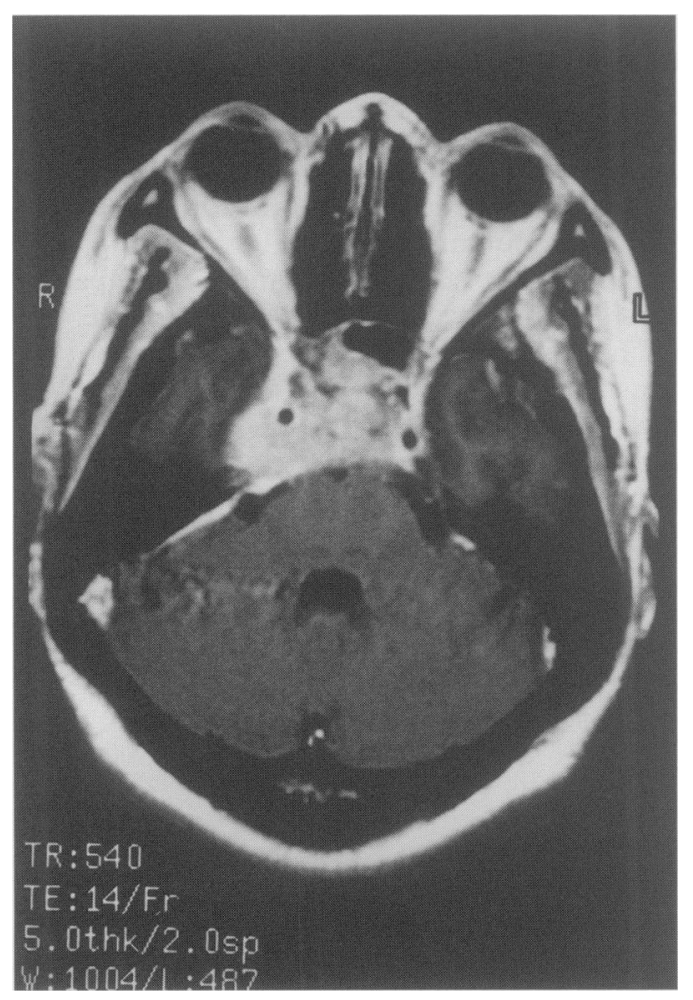

Figure 1 T1-weighted MRI scan

\section{Questions}

1 Where was the lesion responsible for the cranial neuropathies?

2 What was the diagnostic procedure?

3 What was the final diagnosis and what are the prognostic implications? 
Answers

\section{QUESTION 1}

The combination of a sixth and trigeminal nerve lesion places the pathology in the region of the right cavernous sinus and this is well demonstrated on the MRI.

\section{QUESTION 2}

The diagnostic procedure was a lumbar puncture which showed a protein of $1.19 \mathrm{~g} / 1$, with a white cell count of 4 cells $/ \mathrm{ml}$, and $1 \mathrm{red}$ cell $/ \mathrm{ml}$. The white cells were spun down and were shown to consist of $49 \%$ plasma cells in various stages of maturity, including nucleoli and mitosis.

\section{QUESTION 3}

The final diagnosis was myeloma relapse presenting as multiple cranial neuropathies. The prognosis is grave, and this patient died after eight weeks.

\section{Discussion}

The combination of trigeminal and sixth nerve palsies strongly suggests cavernous sinus disease due to a tumour (most commonly secondary deposits due to breast or lung cancer) or intracavernous aneurysm. Rarely, inflammatory causes such as granulomatous disease or chronic fungal infection are to blame. The MRI shows a large mass invading the right cavernous sinus. The tissue diagnosis was made by detecting plasma cells in cerebrospinal fluid examination.

Cranial neuropathy due to myeloma is a rare complication of multiple myeloma. ${ }^{1-4}$ Nerve

1 Clarke E. Cranial and intracranial myeloma. Brain 1954; 77: $61-81$.

2 Cushing H. Intracranial tumours. Notes upon a series of two thousand verified cases with surgical mortality percentages pertaining thereof. Illinois: Springfield, 1932.

3 Silverstein A, Doniger DE. Neurological complications of myeloma. Arch Neurol 1963; 9: 102-13. palsies are generally produced by myeloma deposits at the base of the skull with the body of the sphenoid and the apex of the petrous bone the commonest sites, with sixth, eighth, and trigeminal nerve palsies being most frequent. Cranial neuropathies have previously been reported as occurring at various stages of the disease, but it is unclear when patients are most susceptible and what the prognostic significance of this complication is. ${ }^{1-4}$ Whilst cranial neuropathies themselves have not in the past been prescient of bad outcome, when they are manifestations of disease relapse they may be harbingers of a poor prognosis when other major treatment modalities have already been used and when associated with evidence of generalised myeloma. Most patients die within six months.

This patient illustrates two important points. Firstly, that cranial nerve involvement in myeloma can be the first manifestation of disease relapse, even when initial investigations suggest otherwise. Secondly, that MRI is the investigation of choice to localise the lesion, and that analysis of cerebrospinal fluid with centrifuge and cytospin is useful to confirm that the lesion visible on MRI is likely to be composed of plasma cells.

\section{Final diagnosis}

Cranial neuropathy as a manifestation of myeloma relapse.

Keywords: multiple myeloma, cranial neuropathy, magnetic resonance imaging, sixth nerve palsy

\footnotetext{
4 Brenner B, Carter A, Tatarsky I, Gruszkiewicz I, Peyser E. Incidence, prognostic significance and therapeutic modalities of central nervous system involvement in multiple myeloma. Acta Haematol 1982; 68: 77-83.
} 